\section{Cupid: A program for computations with probability distributions}

\author{
JEFF MILLER \\ University of Otago, Dunedin, New Zealand
}

This article describes Cupid, a program that performs computations with univariate probability distributions. Cupid can be useful in stochastic modeling and simulation hypothesis testing, and examination of statistical procedures. The program runs on IBM-PC compatibles under the DOS operating system, and it is free for educational and noncommercial use.

Behavioral researchers are often interested in developing quantitative models of stochastic mechanisms, but few computer programs are available to facilitate this process. Probability and statistics books define many probability distributions that could serve as plausible models (e.g., Johnson, Kotz, \& Balakrishnan, 1994), but it can be inconvenient to compute predicted values from a given theoretical distribution. As one example, researchers attempting to model full distributions of reaction time (RT) - rather than simply mean and variance-would have to carry out numerical integration to compute the predicted RT at a given percentile from, say, a log normal or exGaussian distribution with a particular set of parameters.

The computer program Cupid, which runs on IBMPC-compatible computers and is freely available for educational and noncommercial use, was written to aid in the development of models involving univariate probability distributions. Table 1 lists the probability distributions defined within the program, and Table 2 lists the predicted values that the program can compute.

Cupid uses a command-line interface that can be driven interactively or in batch mode. The user tells the program which distribution to use by typing its name and parameters, and then the user asks Cupid to compute specific predicted values within that distribution. For example, the user would enter the following three commands to select the gamma distribution with parameters of three and .01, and then to compute this distribution's kurtosis and its percentile points corresponding to the percentiles of 5,15 , $25, \ldots, 95$ :

\section{Gamma(3,.01) \\ Kurtosis \\ Repeat(Pctile,.05,.1,.95)_InverseCDF (Pctile)}

The third command illustrates Cupid's iterative capabilities: The function InverseCDF (Pctile) is computed re-

Correspondence should be addressed to J. Miller, Department of Psychology, University of Otago, Dunedin, New Zealand (e-mail: miller@otago.ac.nz). peatedly, with the variable Pctile taking on values from 0.05 to 0.95 by increments of 0.1 .

In addition to the basic distributions shown in Table 1, Cupid has the ability to form many kinds of derived distributions. For example, one can form a new distribution by truncating one of the basic random variables (i.e., restricting it to a certain range), or by transforming it with a linear, inverse, exponential, power, or log transform. One can also form a derived distribution from two or more underlying distributions, by taking a convolution, a mixture, or an order statistic involving the underlying random variables. Because the program is fully recursive, one can nest these operations (e.g., form a convolution of a mixture distribution and a power transform). Once the desired derived distribution is specified, the commands in Table 2 can be used to compute its properties.

Cupid can do a number of useful things with both basic and derived distributions, in addition to computing the values shown in Table 2. First, it can generate random numbers, for use in simulations. Second, it can display plots of regular and cumulative probability distribution functions, hazard functions, and moment-generating functions. These plots use low-resolution character-based graphics (approximately $70 \times 20$ ) but are adequate for visual inspection in most cases. Third, it can estimate parameters, using a simplex-based algorithm (Rosenbrock, 1960). For example, it can adjust the parameters of a distribution to give a desired mean and standard deviation, to maximize the likelihood of a data set, or to yield a desired set of percentile points. In some cases, however, it is necessary to start the parameter estimation algorithm with values not too far from the correct solution in order for it to converge properly.

Cupid can be useful in hypothesis testing as well as stochastic modeling. For example, it may be used to obtain critical values for test statistics with any desired parameter values (e.g., critical value of Pearson's $r$ with $118 d f$ ), eliminating the need for interpolation. If desired, in fact, one can easily generate quite complete tables. For example, the following command would generate a complete $1,000 \times 1,000$ table of critical $F$ values for the .05 signif-

Table 1

List of Cupid's Basic Probability Distributions

\begin{tabular}{ll} 
Beta & Logistic \\
Binomial & Log normal \\
Cauchy & Normal \\
Chi-square & Pearson's $r$ \\
Ex-Gaussian & Poisson \\
Exponential & Rayleigh \\
Extreme value & Studentized range \\
$F$ (Fisher's) & Student's $t$ \\
Gamma & Triangular \\
Laplace & Uniform \\
Lilliefors & Weibull \\
$A^{\prime} \& d^{\prime}$ (from signal detection theory) & \\
\hline
\end{tabular}


Table 2

Partial List of Values That Cupid

Can Compute for Each Distribution

Probability density and cumulative distribution functions

Inverse cumulative probability

Mean, standard deviation, variance, skewness, and kurtosis

Random numbers

Log likelihood of a set of observations

Moments: Raw, central, and conditional

Hazard and moment-generating functions

icance level with all degrees of freedom in the range of 1 to 1,000 for both numerator and denominator, if that were desired:

$$
\begin{aligned}
& \text { Repeat(df1,1,1,1000)_Repeat(df2,1,1,1000) } \\
& \text { _F (df1,df2)_InverseCDF }(0.95)
\end{aligned}
$$

Two of the distributions defined within Cupid are of particular interest to psychophysicists. Specifically, Cupid will tabulate the distributions of the signal detection theoretic measures $d^{\prime}$ and $A^{\prime}$ for the yes-no task for an experiment with a given number of trials, hit rate, and false alarm rate (Green \& Swets, 1966). These tabulations yield the critical values needed to test a null hypothesis about the true value of one of these parameters $\left(d^{\prime}\right.$ or $\left.A^{\prime}\right)$ for a given subject and to compute confidence intervals for the true values, given the data set (Miller, 1996).

Because Cupid is such a general program, it may be helpful to see a few specific examples of how it could be used. Three examples follow:

1. An RT researcher wanting to evaluate the effectiveness of a proposed new method for eliminating outliers might use Cupid to generate random samples of RTs for a simulation study. RTs could easily be generated from distributions including outliers (e.g., a mixture of an exGaussian with a mean of $350 \mathrm{msec}$ and a normal with a mean of $2 \mathrm{sec}$ ), as well as from distributions excluding outliers. As this example illustrates, Cupid is among other things a toolbox of random number generators. Cupid's ability to run in batch mode would be especially useful in this example, because each iteration of the simulation process would involve one run of Cupid to generate a sample of RTs and one run of an analysis program to compute the desired statistics from the sample.

2. A researcher is interested in evaluating the distributional fit of a given set of observations to various prob- ability distributions (e.g., gamma, log normal, normal, etc.). For any given distribution, Cupid will search for maximum-likelihood estimates of the distribution's parameters, and will report the log likelihood of the data set with those parameter values. The researcher can easily try various distributional families to see which one yields the highest likelihood of the given observations.

3 . A researcher would like to compute a $95 \%$ confidence interval for the true probability of a success $(p)$, given a binomial sample of 100 observations including four successes. The normal approximation is not appropriate because of the small probability of success, but Cupid can be used for exact computations. The lower bound of the confidence interval can be obtained by asking Cupid to estimate the value of $p$ for which the sample would contain three or fewer successes $97.5 \%$ of the time - a value of $p$ that turns out to be .011 . Values of $p$ smaller than .011 can be rejected (i.e., lie outside the confidence interval consistent with this sample), because they predict that four or more successes would be observed in $2.5 \%$ of samples or less. Analogously, the upper bound can be obtained by asking Cupid to estimate the value of $p$ for which four or fewer successes would be observed only $2.5 \%$ of the time, resulting in an upper bound of $p=.09926$.

In addition to the program file Cupid.exe, Cupid comes with a postscript file containing a printable manual and an ASCII version of the manual that can be inspected on line. The complete version can be obtained by sending a formatted 3.5-in. high-density floppy disk and a stamped, self-addressed mailer to the author, and it is also available by ftp from common archive sites in the file Cupid1__1.Zip (the numbers in the file name may change as future versions are developed).

\section{REFERENCES}

GREEN, D. M., \& SwETS, J. A. (1966). Signal detection theory and psychophysics. New York: Wiley.

Johnson, N. L., KotZ, S., \& BalakrishnaN, N. (1994). Continuous univariate distributions. New York: Wiley.

Miller, J. (1996). The sampling distribution of $d^{\prime}$. Perception \& Psychophysics, 58, 65-72.

RoSENBROCK, H. H. (1960). An automatic method for finding the greatest or least value of a function. Computer Journal, 3, 175-184.

(Manuscript received October 28, 1996; revision accepted for publication January $29,1997$. ) 